\title{
A Multilevel Subgridding Scheme for Two-Dimensional Finite-Difference Time-Domain Method
}

\author{
Ching-Cheng Chang and Shyh-Kang Jeng \\ Graduate Institute of Communication Engineering and \\ Department of Electrical Engineering \\ National Taiwan University \\ Taipei, Taiwan, ROC \\ Email: skjeng@ew.ee.nn.edu.tw
}

\begin{abstract}
Finite-Difference Time-Domain Method (FDTD) has being very popular in solving electromagnetic problems. However, for those that contain small structures or strongly varying fields, very fine meshes have to be used. Since a finer mesh means longer computing time and larger memory, subgridding would be one of the ways to improve the performance of the FDTD method. To expand the power of subgridding, we develop a multilevel subgridding scheme. This new scheme can provide smoother transitions than the conventional one-level subgridding scheme, and achieve better performance.
\end{abstract}

Introduction

A subgridding scheme divides the problem into regions with different grid sizes. Usually the grid size in coarse region is $\lambda 10 \sim \lambda 20$ as in normal FDTD, and this size is scaled by an integer ratio in the fine region. For previous studies on subgridding [1]-[3], there's usually only one subgrid region. If the resolution is not enough, a higher ratio of grid sizes for subgridding would be needed. It will take longer computing time and may cause more error due to more serious mismatch of grid sizes. A multilevel subgridding scheme can divide grids by a small grid-size ratio between adjacent level to simplify programming and reduce the error owing to the mismatch of grid size.

Multilevel Subgridding Scheme

A multilevel subgridding scheme is shown in Figs. 1 and 2. Here the ordinary FDTD and subgridding method is as the core process. For a 2-D TM FDTD problem, a coarse 
grid is divided by a factor of 3 , and a second-order homogeneous traveling wave equation is used for spatial interpolation to find out the extra information at the boundary of a subgrid region. During the transition between grids of adjacent levels, linear interpolation in time and bilinear interpolation in space are used. And for updating the $\mathrm{H}$ fields at the boundary of a upper grid, the data are sampled at the common $\mathrm{H}$ field points located at the boundaries of the lower-level subgrid region.

Further, a tree-like data structure is used in which a flag is added to tell if the grid of a certain level is divided into subgrids or not. As the algorithm shown in Fig. 2, the calculation is designed to be recursive. First the calculation starts at the very lower-left main cell and scans all cells row by row. While scanning all cells, the program will determine if this is a grid with a subgrid. Once the program finds that the cell being dealt with has a subgrid, it starts another FDTD procedure for this subgrid region. If the cell is not subdivided, a normal iteration process of FDTD is applied. Every subgrid region basically is treated as a normal FDTD case, where the process starts at the grid with index $(i, j)=(0,0)$ in the local index system and has boundary conditions defined by interaction of regions in adjacent levels. It also identifies if there is any other subgridding of next level while doing iteration. This process repeats until all cells in the region have been calculated. The control is then returned to the previous level.

\section{Numerical Example}

For verification, a PEC cylinder with a current filament in free space shown in Fig. 3 is solved. The frequency of the current source is $1 \mathrm{GHz}$ and the main grid size is $0.015 \mathrm{~m}$. The test region is divided into $251 \times 201$ main cells with a $/ 20$ mesh size. At the border of the test zone, the second-order Mur's absorbing boundary condition is applied. Four different schemes were tested and compared. One scheme is with uniform coarse grid with cell size $\Delta=15 \mathrm{~mm}$, and one is with uniform fine grid in $1 / 3$ main cell size. The other two schemes are with subgrids: one is with a level of subgrid, in which the subgrid size is $\Delta / 3$; and the other is with two levels of subgrids as shown in Fig. 4 . The size of cells in the second level of the two-level scheme is $\Delta / 9$.

The curves in Fig. 5 report the steady state total field which is the response after about 27 periods around a circle of radius $0.6 \mathrm{~m}$ outside the cylinder. Comparisons of different schemes are given in Table I. The one-level subgridding scheme works well as in other reports. The two-level scheme improves the accuracy even more, and takes only $70 \%$ of the time in the uniform fine grid scheme. Moreover, for a single round of simulation, the two-level subgridding already offers 9 times the resolution of the main 
cell, and 3 times the resolution of the uniform fine grids within a comparable short time.

\section{Conclusion}

A multilevel subgridding scheme has been developed. It has been shown that it can improve the resolution of FDTD. Though we only apply it for a 2D case here, it can be easily extended for general 3D problems.

\section{Reference}

[1] Svetlana S. Zivanovic, Kane S. Yee, and Kenneth K. Mei, "A Subgridding Method for the Time-Domain Finite-Difference Method to Solve Maxwell's Equations," IEEE Trans. Microwave Theory Tech., vol. 39, No.3, pp.471-479, March 1991.

[2] Michael W. Chevalier, Raymond J. Lubbers, and Vaughn P. Cable, "FDTD Local Grid with Material Traverse," IEEE Trans. Antenna Prop., vol. 45, No.3, pp.411-421, March 1997.

[3] Wenhua Yu, and Raj Mittra, "A New Subgridding Method for the Finite-Difference Time-Domain (FDTD) Algorithm," Microwave and Optical Tech. Lett., vol. 21, No.5, pp.330-333, June 1999.

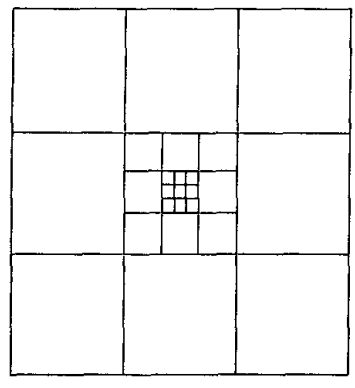

Fig. 1 Multilevel subgridding

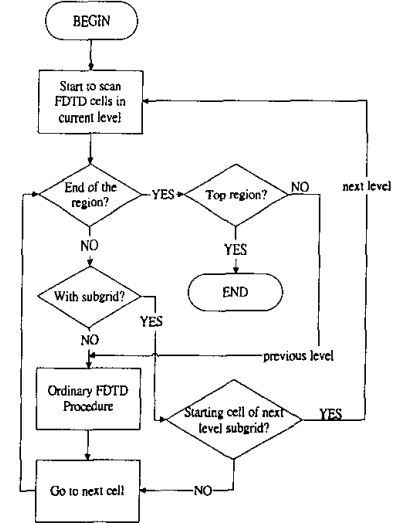

Fig. 2 The recursive algorithm for multilevel subgridding 


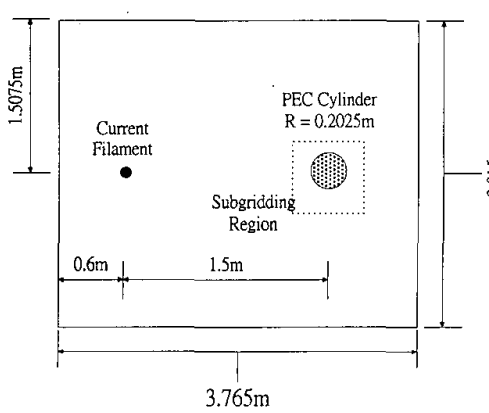

Fig. 3 A PEC cylinder with a current filament

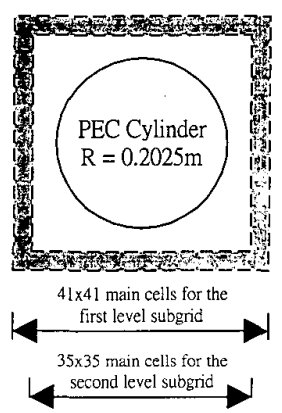

Fig. 4 Range of subgridding

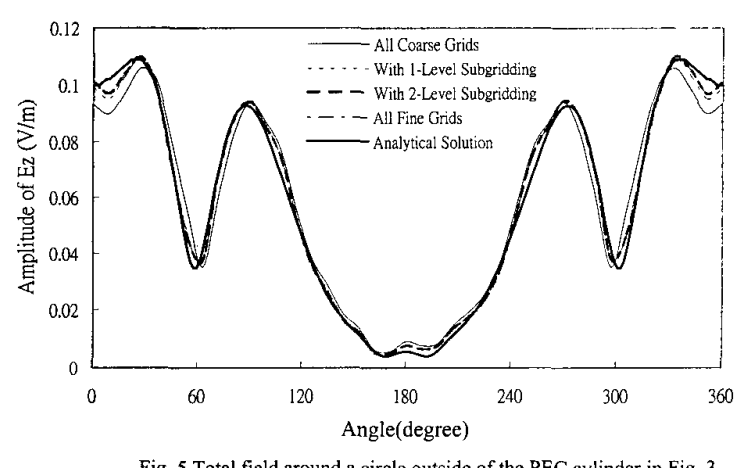

Fig. 5 Total field around a circle outside of the PEC cylinder in Fig. 3

\begin{tabular}{|c|c|c|c|c|c|}
\hline Scheme & Cell size & Time step & CPU time & Cell numbers & $\begin{array}{c}\text { Root mean square } \\
\text { error }\end{array}$ \\
\hline $\begin{array}{c}\text { uniform coarse } \\
\text { grid }\end{array}$ & $\Delta=15 \mathrm{~mm}$ & $\Delta \mathrm{t}=0.025 \mathrm{~ns}$ & $481.090 \mathrm{~s}$ & 51359 & $6.502 \mathrm{e}-3$ \\
\hline $\begin{array}{c}\text {-level } \\
\text { subgridding }\end{array}$ & $\Delta, \Delta / 3$ & $\Delta \mathrm{t}, \Delta \mathrm{t} / 3$ & $860.730 \mathrm{~s}$ & 66984 & $3.184 \mathrm{e}-3$ \\
\hline $\begin{array}{c}2 \text {-level } \\
\text { subgridding }\end{array}$ & $\Delta, \Delta / 3, \Delta / 9$ & $\Delta \mathrm{t}, \Delta v 3, \Delta t / 9$ & $8486.15 \mathrm{~s}$ & 151848 & $2.670 \mathrm{e}-3$ \\
\hline $\begin{array}{c}\text { uniform } \\
\text { finc grid }\end{array}$ & $\Delta / 3$ & $\Delta v / 3$ & $12257.7 \mathrm{~s}$ & 456775 & $2.670 \mathrm{e}-3$ \\
\hline
\end{tabular}

Table I Performance of different schemes for the problem shown in Fig. 3. For every scheme 1500 coarse-grid time steps are requested 